\title{
Surveillance of the emerging enterovirus D68 in Canada: An evaluation
}

\author{
Reyes Domingo $\mathrm{F}^{1 \star}$, McMorris $\mathrm{O}^{1}$, Mersereau $\mathrm{T}^{1}$
}

\begin{abstract}
Background: In the fall of 2014, in response to outbreaks of an emerging respiratory pathogen enterovirus D68 (EV-D68) which affected mostly children, a rapid time-limited surveillance pilot for hospitalized cases was conducted in seven Canadian jurisdictions.
\end{abstract}

Objective: To evaluate whether the goals of the EV-D68 pilot were met and to determine the benefits of and lessons learned from a rapid-response surveillance system for emerging pathogens.

Methods: An evaluation survey was created and administered via a secure online link. All provinces and territories (PTs) and federal partners involved in the pilot were invited to complete one survey per jurisdiction $(\mathrm{N}=17)$. Proportions were calculated for responses to closed-ended questions and recurring themes were identified for open-ended questions.

Results: Fifty four percent (7/13) of PTs and $50 \%(2 / 4)$ of federal partners completed the survey. All four goals of the pilot were met to some degree. All respondents agreed that there were important benefits to rapid surveillance initiatives for emerging pathogens including the capacity to: better understand the epidemiological and clinical features as well as the public health risk of emerging pathogens (66.7\%); inform public health action (66.7\%); collaborate and avoid duplication of work (11.1\%); test and develop jurisdictional capacity (11.1\%); and inform future response efforts (11.1\%). Receiving timely case summaries (preferably weekly) was identified as important for $88 \%$ of respondents. In terms of lessons learned, more than half of respondents (66.7\%) indicated that current processes needed to be improved in order to facilitate rapid surveillance initiatives within and across jurisdictions including the need to develop data-sharing agreements and have pre-existing protocols. Important factors identified for a surveillance data reporting platform included: ease of functionality, data security, jurisdictional control, web-based and flexibility to meet changing surveillance needs.

Conclusion: Evaluation results from the EV-D68 surveillance pilot will assist with future rapid surveillance initiatives. It is important that lessons learned be addressed prior to the emergence of the next emerging pathogen.

\section{Affiliations}

${ }^{1}$ Centre for Immunization and Respiratory Infectious Diseases, Public Health Agency of Canada, Ottawa, ON

\section{*Correspondence}

francesca.reyesdomingo@phacaspc.gc.ca

Suggested citation: Reyes Domingo F, McMorris O, Mersereau T. Surveillance of the emerging enterovirus D68 in Canada: An evaluation. Can Comm Dis Rep 2016;42:4-8. https://doi.org/10.14745/ccdr.v42i01a01

\section{Introduction}

Enterovirus D68 (EV-D68) is a non-polio enterovirus that often causes mild symptoms such as a cold or fever; however more severe respiratory symptoms such as difficulty breathing or wheezing may be reported in individuals, particularly children with a history of asthma or other pre-existing conditions (1). Prior to 2014, EV-D68 was rarely identified in Canada. The National Microbiology Laboratory (NML) identified only 85 EV-D68 positive isolates from 1990 to early 2014 (2).

In the fall of 2014, there was a large outbreak of EV-D68 virus in Canada, the United States $(3,4)$ and elsewhere. The outbreak in Canada caused severe respiratory illness requiring hospitalization in over 200 children (3) and resulted in several deaths (5) as well as cases with neurological symptoms $(3,5)$.

\section{National surveillance of emerging respiratory infections}

The administration and delivery of health care services in Canada is the responsibility of each province and territory (PT) (6), therefore federal/provincial/territorial collaboration and data sharing are required to obtain national surveillance information. There are challenges in developing a national surveillance system for emerging pathogens. Although novel avian influenza and severe acute respiratory syndrome (SARS) are now nationally notifiable (7), in general, reporting mechanisms for national 
surveillance of emerging pathogens are neither available nor well established. In addition, building any surveillance system requires effort and investment ( 8 ) and this can be difficult to do the midst of an outbreak, as demonstrated during the 2003 outbreak of SARS in Canada (9).

\section{EV-D68 severe outcomes surveillance pilot}

Prior to the 2014 outbreak, illness due to EV-D68 was rare and the disease was not nationally notifiable. In the fall of 2014, concerns over initial reports of severe respiratory illness in EV-D68 cases in Canada, combined with a lack of clinical and epidemiological information on EV-D68 in general, prompted the Public Health Agency of Canada (PHAC), in collaboration with select federal and PT partners, to launch a rapid response surveillance pilot to collect data for national reporting within weeks of the outbreak. All 13 PTs were invited to participate in the time-limited rapid surveillance pilot and seven agreed: British Columbia, Alberta, Ontario, New Brunswick, Yukon, Northwest Territories and Nunavut. With this, all five regions in Canada (i.e. Atlantic, Central, Prairies, Western and the Territories) and three of the four most populous provinces were represented. In addition, PHAC's Centre for Immunization and Respiratory Infectious Diseases (CIRID), the NML, the Canadian Field Epidemiology Program and Health Canada's First Nations and Inuit Health Branch were also involved in the pilot.

The goals of the EV-D68 pilot were to: (a) collect select de-identified case-level data on severe cases of EVD68 infection in Canada requiring hospitalization; (b) describe select clinical and epidemiological characteristics of severe cases of EV-D68 infection in near-real time and at the end of the surveillance period; (c) provide participating jurisdictions with options for creating real-time summary reports (e.g, summaries of national or jurisdiction-specific data); and (d) establish a secure web-based reporting tool that could be adapted for future surveillance needs. Data on hospitalized, laboratory-confirmed cases of EV-D68 were collected from September to October 2014. A surveillance summary of pediatric cases $(<=18$ years of age) for the month of September 2014 was published in February 2015 (3). An evaluation of the pilot followed. The overall objectives for the evaluation of the EV-D68 pilot were to: (a) assess whether the goals of the EV-D68 pilot were met, (b) determine the benefits of a rapid-response surveillance system for emerging pathogens and (c) identify valuable lessons from the pilot process for future surveillance efforts.

\section{Methods}

PHAC conducted a qualitative evaluation of the EV-D68 pilot in the spring of 2015. The survey questions were created by the authors and developed specifically to determine whether the goals of the EV-D68 pilot were met, to determine the benefits and to identify lessons learned by articulating what worked or did not work well, including barriers or limitations that were identified throughout the surveillance pilot and what could be done to address them. (The full survey is available upon request from the corresponding author.) The survey was pilottested for clarity and comprehensibility by two individuals from CIRID who were not involved in the EV-D68 pilot. Respondents were reminded of which goals of the pilot the specific survey questions were applicable to and were provided with supplementary information for reference (e.g., link to the final pilot report, reminder of events/circumstances that occurred). The survey was available in English and French and administered through FluidSurveys ${ }^{\mathrm{TM}}$ (10).

Surveys were sent to the emerging pathogens surveillance leads in all PTs $(n=13)$ and four branches or centres within the two federal departments involved in the pilot. Jurisdictions were encouraged to complete one survey each but the initial recipients could forward the survey to other individuals as appropriate. Survey respondents were informed that if multiple survey responses per jurisdiction were received, responses would be weighted appropriately so as not to bias the results. The individuals involved in planning and administrating the evaluation and those at the Canadian Network for Public Health Intelligence (CNPHI) who created the data reporting platform, did not participate in the survey as they were not among the target population. Two e-mails and two verbal reminders were sent to increase the survey's participation rate.

A variety of survey question types (e.g., multiple choice, select all that apply, open-ended) were asked. Proportions were calculated for responses to close-ended questions; recurring themes were identified from open-ended questions for which similar responses were grouped together and percentages applied accordingly. Unique responses were summarized and listed in qualitative form in the results.

Reasons for not participating in the pilot were gathered throughout the pilot process from the PTs that declined to participate (verbally during pre-scheduled EV-D68 pilot teleconferences, at the monthly respiratory surveillance teleconferences or in writing via e-mail).

\section{Results}

\section{Participation}

Fifty-four percent (7/13) of PTs and 50\% (2/4) of the federal partners completed the evaluation survey. Of the respondents, all but one jurisdiction participated in the surveillance pilot. Respondents included, but were not limited to: the four jurisdictions with the largest populations in Canada and subsequently the same jurisdictions that reported the most EV-D68 cases in the fall of 2014; federal and territorial jurisdictions responsible for northern and/or Aboriginal populations; and one province from the Atlantic region. The three provinces that did not complete the survey and did not participate in the pilot provided their reasons via email.

Those jurisdictions/organizations that participated in the pilot did so because they wanted to determine the burden of EV-D68 nationally (87.5\%); the virus was an emerging pathogen of public health interest (25\%); and/or they wanted to better understand processes and working relationships between multijurisdictional partners when novel communicable diseases occur (25\%). One survey respondent noted he/she did not participate in the pilot because EV-D68 was not a notifiable disease. The three provinces who did not complete the survey and did not participate in the pilot indicated via e-mail that the primary reason for not participating was due to limited capacity and resources within their province. At that time, some provincial 
public health laboratories did not have the capacity to test for EV-D68 or had limited provincial human resources available to participate.

\section{Overall experience}

The official start of the pilot occurred three weeks after PHAC was notified of the first EV-D68 case in Canada. When asked, $75 \%$ of respondents indicated they were 'satisfied' or 'very satisfied' with the length of time it took to develop the pilot and initiate surveillance.

The active surveillance period lasted six to eight weeks and included retrospective case ascertainment for the month of September. The larger jurisdictions reported the majority of EV-D68 cases while few to none were reported from the smaller jurisdictions. Despite the variation in effort required per jurisdiction to collect case data, $62.5 \%$ found the amount of time and effort required to participate in the pilot was 'somewhat reasonable' and 25\% found it 'very reasonable'. Overall, $62.5 \%$ found that participation in the pilot was 'somewhat satisfying'. All respondents indicated their jurisdiction/organization were provided with regular updates on the status throughout the pilot.

\section{Goals of the surveillance pilot}

The first goal was to collect select de-identified case-level data on severe cases of EV-D68 infection in Canada requiring hospitalization. All participating PTs $(n=7)$ agreed to use the case report form provided. An accompanying web-based electronic reporting platform (developed by CNPHI) was used to collect data. However, only four of the PTs were able to identify at least one case that met the case definition and thus, only these four PTs were able to report case data.

The second goal was to describe select clinical and epidemiological characteristics of severe cases of EV-D68 infection in near-real time and at the end of the surveillance period. An epidemiological summary of the cases was provided at the end of the surveillance period. A published surveillance summary report included case data from three of the seven participating PTs and was limited to hospitalized pediatric cases $<18$ years who were laboratory-confirmed in the month of September (3). Despite the analyses being limited to data from a small number of jurisdictions and regarding children, $62.5 \%$ of respondents found the summary report provided was 'useful' or 'very useful'. For those who found it less useful (37.5\%), the main reason was because the summary report was not representative of the national picture, particularly due to limitations in the geographic and age distributions of the cases included.

The third goal was to provide participating jurisdictions with options for creating real-time summary reports via CNPHI (e.g., summaries of national or jurisdiction-specific data). These were not provided because, of the jurisdictions that reported at least one case ( $n=4$; all of whom completed the survey), only $50 \%$ used the case report form and only $25 \%$ used the CNPHI platform to report case information nationally.

The fourth goal was to establish a secure web-based reporting tool that could be adapted for future surveillance. Although the CNPHI platform had the capability to provide participating jurisdictions with options to create real-time summary reports, was secure, web-based and had the flexibility to adapt to user needs (e.g., allow for batch data uploads rather than manual data entry for each case, addition/deletion of data fields, etc.), uptake during the pilot was low. Some jurisdictions did not use the case report form or the CNPHI platform because they either used their own jurisdiction-specific forms or databases or they did not want to place additional burden on limited health care resources.

\section{Benefits of a rapid surveillance initiative for emerging pathogens}

All respondents indicated there was a need for rapid surveillance initiatives for emerging pathogens. Their reasons were to better understand epidemiological and clinical features and public health risk of the emerging pathogen (66.7\%); to inform public health action (66.7\%); to collaborate with partners and avoid duplication of work (11.1\%); to test and develop jurisdictional capacity $(11.1 \%)$; and because lessons learned may guide future preparation and response efforts (11.1\%).

\section{Lessons learned}

More than half of respondents $(66.7 \%)$ indicated that current processes should be improved in order to facilitate rapid surveillance initiatives within and across jurisdictions. Specifically, there should be more opportunities to engage in or practice rapid surveillance initiatives; ethics approvals should be expedited to enable timely data collection; and data reporting formats and mechanisms should be flexible to prevent duplication of, or avoid unnecessary effort and use of resources.

Half of the respondents indicated that data-sharing agreements or protocols should be established prior to an outbreak. For example, ensure that emerging pathogens showing epidemic or unusual features are reportable conditions under PT communicable disease regulations; ensure data-sharing agreements are in place that promotes sharing of data and support collaborative opportunities; as well as pre-existing national protocols for coordinating the response to these pathogens. Finally, 33.3\% of respondents indicated that in order to facilitate rapid surveillance initiatives within and across jurisdictions, jurisdictions need to ensure that sufficient resources and staffing (e.g., surge capacity) are in place to respond to an emerging public health threat.

The majority of respondents (88\%) indicated that receiving timely case summaries from PHAC throughout the pilot process was important and weekly updates were preferred. Opportunities to discuss outbreak findings and any concerns with those participating in the pilot on a regular basis were suggested.

Respondents selected the following characteristics as important in a data reporting platform: ease of functionality (100\%); security of data storage and access to data (87.5\%); ability to upload batch data (87.5\%); ability to maintain jurisdictional control for data access and reporting (87.5\%); web-based (75\%); and the flexibility to rapidly create case report forms for new surveillance initiatives (62.5\%).

Finally, $40 \%$ of participants suggested that barriers be identified and addressed prior to an emergent infectious disease outbreak. 
For example, jurisdictions should establish mechanisms to quickly collect and report data, including data about diseases that are not necessarily reportable or notifiable. Forty percent also suggested that protocols should be established with roles and responsibilities clearly outlined for multi-jurisdictional surveillance processes. Twenty percent suggested that an interjurisdictional group could assess the need for and decide on whether to proceed with setting up a new surveillance system. A similar proportion suggested that from the outset, jurisdictions should place a priority on setting aggregate reporting of surveillance data to ensure timeliness of data reporting because case-level data reporting requires privacy considerations which take additional time and resources to collect.

A summary of the key evaluation findings are noted in Table 1.

\section{Discussion}

The key findings of the evaluation of the EV-D68 surveillance pilot were that: the four goals of the pilot were met albeit with some shortcomings; there were important benefits to conducting surveillance of emerging pathogens; and some valuable lessons were learned that could assist with future surveillance initiatives for emerging pathogens in Canada. The survey findings highlighted the following important success factors: pre-established processes and reporting mechanisms; and adequate resource capacity (particularly human resource surge capacity for additional surveillance demands). Because barriers to surveillance and reporting of pertinent case details caused delays and/or prevented jurisdictions from participating, it is recommended that barriers be identified and addressed prior to the emergence/re-emergence of respiratory pathogens to ensure timeliness in initiating such systems.
Although the surveillance pilot objectives were met, the evaluation was able to shed light on the factors that influenced the level of success achieved for each objective. For example, although a surveillance reporting platform with the ability to provide real-time summary reports was created and made available for PTs to use, uptake of the platform was sub-optimal due to preferences in using jurisdiction-specific forms/platforms. At the federal level, it is important to understand the factors that allow PTs to participate in a national surveillance initiative and what factors sustain national reporting. For example, PHAC could provide forms and reporting mechanisms that minimize undue effort and demands on already-strained PT resources.

The evaluation results should be interpreted with caution due to several limitations. First, close to half of the PTs did not participate in the surveillance pilot or complete an evaluation survey and therefore, only minimal information could be obtained on the usefulness of conducting surveillance on emerging pathogens such as EV-D68 from those jurisdictions. Most of the responses/results came from jurisdictions that participated in the pilot and depending on whether some questions were applicable to a jurisdiction or not, the denominators for percentage calculations were even smaller (i.e. fewer than five). Although non-participating jurisdictions were asked to provide their reasons for not participating, overall, feedback from nonparticipating jurisdictions was lacking. Secondly, the evaluation was conducted five months after completion of the pilot to coincide with a downturn in respiratory virus activity in the country, so as not to burden jurisdictions with the additional task of completing the survey during the active respiratory surveillance period. More complete and informative feedback may have been obtained if the survey was conducted within weeks rather than months following the completion of the pilot.

\section{Table 1: Summary of key evaluation findings of the EV-D68 surveillance pilot}

\begin{tabular}{|c|c|}
\hline $\begin{array}{l}\text { Were the goals of the EV-D68 } \\
\text { surveillance pilot met? }\end{array}$ & $\begin{array}{l}\text { 1. Were select case-level data collected? Yes, but only four of the participating PTs were able to identify at } \\
\text { least one case that met the case definition. } \\
\text { 2. Was the clinical and epidemiologic picture of cases described in near-real time and at the end of the } \\
\text { surveillance period? Not in near-real time but at the end of the pilot, of which } 62.5 \% \text { of respondents } \\
\text { found the summary report useful. } \\
\text { 3. Were options for creating real-time summary reports provided? Yes, however these features were not } \\
\text { utilized due to low uptake of the electronic reporting platform. } \\
\text { 4. Was a secure web-based reporting tool that is amenable to adaptation for future surveillance needs } \\
\text { established? Yes, however uptake was low (only } 25 \% \text { used the CNPHI platform). }\end{array}$ \\
\hline $\begin{array}{l}\text { What were the benefits of } \\
\text { conducting a rapid surveillance } \\
\text { initiative for emerging pathogens? }\end{array}$ & $\begin{array}{l}\text { - To better understand epidemiological and clinical features and public health risk of the emerging } \\
\text { - } \text { pathogen }(66.7 \%) \text {. } \\
\text { - To collaborate with partners and avoid duplication of work }(11.1 \%) \text {. } \\
\text { - To test and develop jurisdictional capacity }(11.1 \%) \text {. } \\
\text { - Lessons learned may guide future preparation and response efforts }(11.1 \%) \text {. }\end{array}$ \\
\hline $\begin{array}{l}\text { What were the lessons learned from } \\
\text { the EV-D68 surveillance pilot? }\end{array}$ & $\begin{array}{l}\text { - Improve current processes to facilitate rapid surveillance initiatives (66.7\%), such as more opportunities } \\
\text { to engage in such initiatives, expedited ethics approvals, flexible data reporting formats, obtaining data- } \\
\text { sharing agreements and draft protocols beforehand. } \\
\text { - Ensure sufficient surge capacity is available (both resources and staff) (33.3\%). } \\
\text { - Have regular opportunities to discuss outbreak findings and any concerns among those participating in } \\
\text { the surveillance initiative. }\end{array}$ \\
\hline
\end{tabular}


The fall 2014 EV-D68 outbreak took place over a brief time period and resulted in a small number of deaths and cases with neurological manifestations. Nevertheless, the EV-D68 surveillance pilot provided PHAC and participating jurisdictions with an opportunity to test resource capacities and processes for emerging respiratory pathogens surveillance and to become better prepared to undertake future rapid surveillance initiatives.

\section{Acknowledgements}

The authors would like to thank all the provinces and territories and federal departments that participated and contributed to the EV-D68 surveillance pilot and those who responded/ contributed to the evaluation.

\section{Conflict of Interest}

None.

\section{Funding}

None.

\section{References}

1. Public Health Agency of Canada. Non-polio enterovirus. Ottawa ON: PHAC; 2015. http://www.phac-aspc.gc.ca/idmi/vhf-fvh/enterovirus-eng.php.

2. Booth TF, Grudeski E, McDermid A. National surveillance for non-polio enteroviruses in Canada: why is it important? Can Commun Dis Rep. Feb 2015;41(S1):11-17.

3. Edwin JJ, Reyes Domingo F, Booth TF, et al. Surveillance summary of hospitalized pediatric enterovirus D68 cases in Canada. Can Commun Dis Rep. Feb 2015;41(S1):2-8.

4. Centers for Disease Control and Prevention. Enterovirus D68. Atlanta GA: CDC; March 2015. http://www.cdc.gov/ non-polio-enterovirus/about/ev-d68.html.

5. British Columbia Centre for Disease Control. Emerging respiratory pathogens. British Columbia Influenza Surveillance Bulletin. Influenza Season 2014-2015, Number12, Week 53. http://www.bccdc.ca/NR/rdonlyres/ F2A8BAAF-B226-4761-9C2B-6CF5C1A77DFC/0/ InfluBulletin_Number12_Week53_201415.pdf.

6. Government of Canada. Provincial/territorial role in health. Ottawa ON: Government of Canada; 2015. http:// healthycanadians.gc.ca/health-system-systeme-sante/cardscartes/health-role-sante-eng.php.

7. Public Health Agency of Canada. Notifiable diseases on-line. Ottawa ON: PHAC; 2015. http://dsol-smed.phac-aspc.gc.ca/ dsol-smed/ndis/index-eng.php.
8. World Health Organization. Communicable disease surveillance and response systems: a guide to planning. Geneva: WHO; 2006. http://www.who.int/csr/resources/ publications/surveillance/WHO_CDS_EPR_LYO_2006_1.pdf.

9. Public Health Agency of Canada. Learning from SARS, Chapter 5 - Building capacity and coordination: national infectious disease surveillance, outbreak management, and emergency response. Ottawa ON: PHAC; 2015. http://www. phac-aspc.gc.ca/publicat/sars-sras/naylor/5-eng.php.

10. FluidSurveys. Ottawa ON: SurveyMonkey Canada; 2015. http://fluidsurveys.com. 